\title{
Application Of Boundary Element Method To Predicting The Aerodynamics Coefficient Of Aircraft With Arbitrary Shape Cross Sections
}

\author{
Wan Weiyu \\ Beijing Electro-mechanical Engineering Institute \\ Beijing, China \\ e-mail: vodkabuaa@yahoo.cn
}

\author{
Li Changwen \\ Beijing Electro-mechanical Engineering Institute \\ Beijing , China \\ e-mail: 13681220376@139.com
}

\author{
Pan Xinghua \\ Beijing Electro-mechanical Engineering Institute \\ Beijing , China \\ e-mail: panxinghua628@qq.com
}

\begin{abstract}
This paper introduces an application of boundary element method for predicting the aerodynamics coefficient of aircraft with arbitrary shape cross sections. Aerodynamics coefficient of noncircular body is evaluated based on this method. Comparing with the method adopted by McDonnell Douglas Astronautics Company, a more accurate prediction is achieved based on the Boundary Element method. Combining this method with Component Build-Up method, the aerodynamics coefficient of aircraft is calculated, which can satisfy the aerodynamics design of concept stage. A surrogate model is developed based on design of experiment and radial basis function, which can improve the efficient of this method significantly.
\end{abstract}

Keywords-boundary element method; noncircular cross section; aerodynamic prediction; surrogate model

\section{INTRODUCTION}

For preliminary aircraft design, the aerodynamic analysis method requires to be fast, low cost and relatively high accuracy. Component build-up method (CBM) which assembles a variety of theoretical and empirical methods is one of the most effective methods for preliminary aerodynamic design of the circular body. However, it is not effective for noncircular body. Computational Fluid Dynamics (CFD) which can achieve high accuracy is adopted in this area, but the computational efficiency is low. Especially for preliminary design, the designers choose the aerodynamic shape from various shapes. If the aerodynamic calculation cost much time, the design period will be extended obviously.

In the 1980s, lots of notable investigations have been done in aerodynamic design of arbitrary missile bodies by McDonnell Douglas Astronautics Company. The investigations demonstrate the linearized potential equation is solved based on Brown's method and Hess\&Smith method respectively, from which aerodynamic forces and moments are calculated. In addition, computer codes named Low Observables Design Synthesis Tool (LODST) are available to calculate arbitrary body aerodynamics.
The boundary element method (BEM) is an effective and powerful numerical method used to solve the partial differential equation, the Kernel Idea of which is the transformation of the integrative domain and the discretization of the boundary. The transformation of integrative domain means transforming the full domain into the boundary, which can reduce the time of calculation. The discretization of the boundary means discretizing the boundary into many nodes, which can transform the integral operation into matrix operation.

This paper will focus on four topics. The methods considered for the calculation of the additional apparent mass of the arbitrary shape body based the BEM is emphasized in the first part. The calculated results are compared with the results of Hess\&Smith method and the experimental data, and the accuracy is discussed in the second part. A approach for the calculation of aircraft aerodynamics is proposed with the combination of BEM and CBM in third part. For the improvement of the calculation efficiency, a surrogate model of the aerodynamics calculation is developed based on design of experiment and radial basis function in the last part.

\section{BOUNDARY ELEMENT METHOD FOR APPARENT AREA CALCUlation}

\section{A. The concept of Apparent Area Calculation}

For a slender body, if the velocity perturbation in all there orthogonal directions is very small, the velocity potential within a sheet of fluid results in Laplace's potential equation.

$$
\Phi_{\text {yy }}+\Phi_{z z}=0
$$

Ward introduced a new application of slender body theory using the concept of added mass, based on which the relationship of the body force and the apparent area is derived.

$$
\begin{aligned}
& Z=\rho V_{\infty}^{2} \beta A_{11} \mid \begin{array}{l}
b \\
a
\end{array} \\
& N=\rho V_{\infty}^{2} \alpha A_{22} \mid \begin{array}{l}
b \\
a
\end{array}
\end{aligned}
$$


$\boldsymbol{Z}$ is the yawing force and $\boldsymbol{N}$ is the normal force. $A_{11}$ and $A_{22}$ is the apparent area of the body section.

From (2) and (3), if the $A_{11}$ and $A_{22}$ is calculated, the body force can be achieved, moreover, the aerodynamics coefficient can also be calculated.

The apparent area terms of two dimensional cross sections are defined

$$
A_{i j}=\oint_{s} \Phi_{i} \frac{\partial \Phi_{i}}{\partial \boldsymbol{n}} d s
$$

So the $A_{11}$ and $A_{22}$ can be express as

$$
\begin{aligned}
& A_{11}=\oint_{s} \Phi_{1} \frac{\partial \Phi_{1}}{\partial \boldsymbol{n}} d s \\
& A_{22}=\oint_{s} \Phi_{2} \frac{\partial \Phi_{2}}{\partial \boldsymbol{n}} d s
\end{aligned}
$$

Where $\Phi_{1}$ is the velocity potential in y axis direction of the body axis system, $\Phi_{2}$ is the velocity potential in $\mathrm{z}$ axis direction of the body axis system, $s$ is the curve of the body section boundary. $n$ denotes the unit vector outward normal to $s$.

Discretizing the boundary of the integral domain $s$, the discretized form of integral (5) (6)are expressed as:

$$
\begin{aligned}
& A_{11}=\sum_{i=1}^{N} \Phi_{1 i} \frac{\partial \Phi_{1 i}}{\partial \boldsymbol{n}} \Delta s_{i} \\
& A_{22}=\sum_{i=1}^{N} \Phi_{2 i} \frac{\partial \Phi_{2 i}}{\partial \boldsymbol{n}} \Delta s_{i}
\end{aligned}
$$

Where $N$ is the number of the boundary nodes, $i$ is the index of the nodes.

From (7) (8), the calculation of $A_{11}$ and $A_{22}$ only requires the value of the $\Phi_{i}$ and $\frac{\partial \Phi_{i}}{\partial n}$ in every boundary node which is governed by Laplace's equation

$$
\begin{aligned}
& \left\{\begin{array}{c}
\Phi_{1 \mathrm{yy}}+\Phi_{1 z z}=0 \\
q=\frac{\partial \Phi_{1}}{\partial \boldsymbol{n}}=-(1,0) \bullet \boldsymbol{n}
\end{array}\right. \\
& \left\{\begin{array}{c}
\Phi_{2 \mathrm{yy}}+\Phi_{2 \mathrm{zz}}=0 \\
q=\frac{\partial \Phi_{2}}{\partial \boldsymbol{n}}=-(0,1) \bullet \boldsymbol{n}
\end{array}\right.
\end{aligned}
$$

So if the Laplace's equation is the solved, the $A_{11}$ and $A_{22}$ will be evaluated.

\section{B. Apparent Area Calculation based on BEM}

The finite element method (FEM) is, in general, a versatile and effective method for solving nonlinear problems. The disadvantages of FEM are that large quantities of data are required to discretize the full domain, which is tremendous time-consuming and memoryconsuming. The boundary element method (BEM) is a approach to overcome the disadvantages of FEM, which can reduce the calculation time significantly. The kernel idea of the BEM is the transformation of the integrative domain and the discretization of the boundary.
Using Green's second theorem, the Laplace's equation (9)(10)can be transformed into the integration form as below

$$
\Phi^{i}+\int_{\Gamma} q^{*} \Phi d \Gamma=\int_{\Gamma} \Phi^{*} q d \Gamma
$$

The (11) demonstrates that the integral domain was transformed from the whole fluid domain into the boundary. Thus the value of the $\Phi^{i}$ in $\left(x_{i}, y_{i}\right)$ of the fluid domain can be expressed in the form of boundary integral.

The discretization of the boundary means discretizing the boundary into segments with many nodes, which can transform the integral operation into matrix operation. Discretizing equation (11) for source i yields the following expression

$$
\frac{1}{2} \Phi^{i}+\sum_{j=1}^{n} \int_{\Gamma_{j}} q^{*} \Phi d \Gamma=\sum_{j=1}^{n} \int_{\Gamma_{j}} \Phi^{*} q d \Gamma(i=1,2, \cdots n)(12)
$$

Define

$$
\begin{aligned}
\hat{H}_{i j} & =\int_{\Gamma_{j}} q^{*} d \Gamma \\
G_{i j} & =\int_{\Gamma_{j}} \Phi^{*} d \Gamma
\end{aligned}
$$

Equation (12) can be simplified into

$$
\frac{1}{2} \Phi^{i}+\sum_{j=1}^{n} \hat{H}_{i j} \Phi_{j}=\sum_{j=1}^{n} G_{i j} q_{j}
$$

Define

$$
H_{i j}=\hat{H}_{i j}+\frac{1}{2} \delta_{i j}
$$

Where $\delta_{i j}$ is the Kronecker function,

$$
\delta_{i j}=\left\{\begin{array}{lc}
1 & i=j \\
0 & i \neq j
\end{array}\right.
$$

Equation (15)becomes

$$
\sum_{j=1}^{n} H_{i j} \Phi_{j}=\sum_{j=1}^{n} G_{i j} q_{j}(i=1,2, \cdots n)
$$

Equation (18) can be expressed in matrix form as

$$
\boldsymbol{H} \boldsymbol{\Phi}=\boldsymbol{G} \boldsymbol{Q}
$$

Since elements of $\boldsymbol{H}$ and $\boldsymbol{G}$ can be calculate by Gauss quadrature, equation (19) can be inverted to obtain $\boldsymbol{\Phi}$, i.e.

$$
\boldsymbol{\Phi}=\boldsymbol{H}^{+} \boldsymbol{G} \boldsymbol{Q}
$$

It is noted that $\mathrm{H}$ is, in general, a singular matrix, the invert of which can be calculated based on generalized inverse method. Notice that the normal velocity component $\frac{\partial \Phi_{i}}{\partial \boldsymbol{n}}$ can be obtained according to (9) (10), so the $A_{11}$ and $A_{22}$ can be calculated.

\section{Comparison of BEM And Hess\&Smith's Method For Noncircular Section}

For small angle of attack and small velocity perturbations, the flow boundary conditions will not be violated, which satisfies the restrictions associated with the slender body theory. Thus the force on the body of the noncircular section aircraft can be evaluated with the calculation of apparent area. The methods for calculating the apparent area adopted by McDonnell Douglas Astronautics Company is Hess\&Smith’s 
method, which has proven reliable and suitable for calculating the apparent area. The source point are located inward the cross section. Calculating the effect of each source point upon the normal velocity of each test point located on the boundary will results in a square influence matrix. The unknown source are calculated from the matrix, which determine the velocity potential at each test point, and (5)(6)is integrated to provide the apparent area.

Table1 provides analytic expression for the apparent areas of four different cross-sections. The numerical result from each equation is compared with the value obtained from the method of BEM and the method of Hess\&Smith respectively.

All computer generated values used 50 test points. Compared with Hess\&Smith's method, the results of BEM are more consistent with the analytic results, which verify the efficiency of BEM.

\section{AERODYNAMICS COEFFICIENT OF AIRCRAFT WITH ARBITRARY SHAPE CROSS SECTIONS PREDICTIONS}

An approach for Aerodynamics coefficient of aircraft with arbitrary shape cross sections predictions is proposed in this section. The Component Build-Up method is referenced in the approach, that calculates the aerodynamics of aircraft components respectively and build them up. The aerodynamics coefficient of non-circular body is calculated based on BEM, and the coefficient of wings and rudders is evaluated based the theoretical and semi-empirical methods. It is noted that there is no experimental data of the interruption coefficient of the body, the coefficient of equivalent area of circular is adopted in this paper.

The lift coefficient and drag coefficient are evaluated based on this approach for aircraft with two different wings respectively. The results are plotted and compared with wind tunnel data in figure 3-1 and figure 3-2.

The results show a good agreement between computed data and wind tunnel data. In fact, there is a very little difference in lift and drag coefficients between computed data and wind tunnel data at angle of attack from $0^{\circ}$ to ${ }^{\circ}$ and the largest different are computed at $-4^{\circ}$ angle of attack. The reason of the difference is caused by the anomalous nose of the aircraft which not satisfies the slender body theory.

\section{SURROGATE MODEL BASED ON LATIN HYPERCUBE TECHNIQUE AND RBF}

\section{A. Latin Hypercube Technique}

The Latin Hypercube technique is a class of experimental designs that efficiently samples large design spaces. The design space for each factor is divided uniformly (the same number of divisions, $\mathrm{n}$, for all factors). These levels are randomly combined to specify $n$ points defining the design matrix (each level of a factor is studied only once).

For example, "Latin Hypercube Configuration for Two Factors with Five Points" illustrates a possible Latin Hypercube configuration for two factors $\left(X_{1}, X_{2}\right)$ in which five points are studied. While not as visually obvious, this concept easily extends to multiple dimensions.

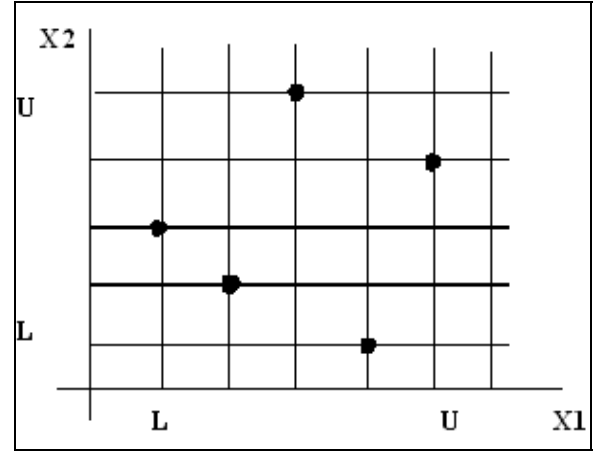

Figure 1. Latin Hypercube Configuration for Two Factors with Five Points

An advantage of using the Latin Hypercube technique over the Orthogonal Arrays technique is that more points and more combinations can be studied for each factor. The Latin Hypercube technique allows the designer total freedom in selecting the number of designs to run (as long as it is greater than the number of factors). A drawback to the Latin Hypercubes is that they are not reproducible because they are generated with random combinations. In addition, as the number of points decreases, the chances of missing some regions of the design space increases.

\section{B. Radial Basis Function}

Radial Basis Function approximation is a type of neural network employing a hidden layer of radial units and an output layer of linear units. RBF approximations are characterized by reasonably fast training and reasonably compact networks. They are useful in approximating a wide range of nonlinear spaces.

Let $x_{1}, \cdots x_{N} \in R^{n}$ be a given set of nodes. Let $g_{j}=g\left(\left\|x-x_{j}\right\|\right) \in R, j=1,2, \cdots N$ be a set of any radial basis functions. Here $\left\|\boldsymbol{x}-\boldsymbol{x}_{\boldsymbol{j}}\right\|$ is the Euclidian distance given by $\left(\boldsymbol{x}-\boldsymbol{x}_{\boldsymbol{j}}\right)^{\boldsymbol{T}}\left(\boldsymbol{x}-\boldsymbol{x}_{\boldsymbol{j}}\right)$. Given interpolation values $y_{1}, \cdots y_{N}$ at data locations $x_{1}, \cdots x_{N}$, RBF interplant

$$
F(x)=\sum_{j=1}^{N} \alpha_{j} g_{j}(x)+\alpha_{N+1}
$$

is obtained by solving the system of $\mathrm{N}+1$ linear equations

$$
\begin{gathered}
\sum_{j=1}^{N} \alpha_{j} g_{j}(x)+\alpha_{N+1}=y_{i}, i=1, \cdots, N \\
\sum_{j=1}^{N} \alpha_{j}=0
\end{gathered}
$$

for $\mathrm{N}+1$ unknown expansion coefficients $\alpha_{j}$.

Hardy (1972, 1990), the primary innovator of the RBF Method, adds a constant to the expansion and constraints the sum of the expansion coefficients to zero as seen in (21)(22), introducing the notation 


$$
\begin{gathered}
\boldsymbol{p}=\left[\begin{array}{c}
1 \\
\vdots \\
1
\end{array}\right] \in R^{N}, \boldsymbol{G}=\left[\begin{array}{ccc}
g_{1}\left(x_{1}\right) & \cdots & g_{N}\left(x_{1}\right) \\
\vdots & \vdots & \vdots \\
g_{1}\left(x_{N}\right) & \cdots & g_{N}\left(x_{N}\right)
\end{array}\right] \in R^{N \times N} \\
\boldsymbol{H}=\left[\begin{array}{cc}
\boldsymbol{G} & \boldsymbol{p} \\
\boldsymbol{p}^{T} & \boldsymbol{0}
\end{array}\right] \in R^{(N+1) \times(N+1)}, \begin{array}{l}
\boldsymbol{\alpha}=\left(\alpha_{1}, \cdots \alpha_{N+1}\right)^{T} \in R^{N+1} \\
\boldsymbol{y}=\left(y_{1}, \cdots y_{N+1}\right)^{T} \in R^{N+1}
\end{array}
\end{gathered}
$$

We can rewrite (22) (23) in the matrix form as

$$
\boldsymbol{H} \boldsymbol{\alpha}=\boldsymbol{y}
$$
by

Then the interpolation expansion coefficients are given

$$
\boldsymbol{\alpha}=\boldsymbol{H}^{-1} \boldsymbol{y}
$$

The derivatives of the interplant can be easily found at the nodes $x_{i}$. For example,

$$
\begin{gathered}
F^{\prime}(x)=\sum_{j=1}^{N} \alpha_{j} g_{j}^{\prime}\left(x_{i}\right), \quad i=1, \cdots, N \\
F^{\prime \prime}(x)=\sum_{j=1}^{N} \alpha_{j} g_{j}^{\prime \prime}\left(x_{i}\right), \quad i=1, \cdots, N
\end{gathered}
$$

Because model physics can vary, a different type of basis function would be needed to provide a good fit. The response surface goes through all the given interpolation data.

\section{Surrogate Model For Aerodynamics Coefficient Prediction}

The surrogate model is a kind of approximation used in place of the original code to facilitate the analysis. The development of the surrogate model requires DOE and approximation methods.

A surrogate model for aerodynamics coefficient prediction is generated based on the Latin Hypercube technique and RBF. Six parameters (span, chord, sweep angle, velocity, altitude and attack of angle) are defined as factors to study. 1000 sample points are selected and calculated based on Latin Hypercube technique for generating the design matrix, then the surrogate model is derived based on RBF. This method is verified by comparing with original model, and the results are shown in Fig. 1. The time cost of the surrogate model is only $1 / 5$ of the original model. The relative error of the drag coefficient is below $7 \%$ and the relative error of the lift coefficient is below $1 \%$.

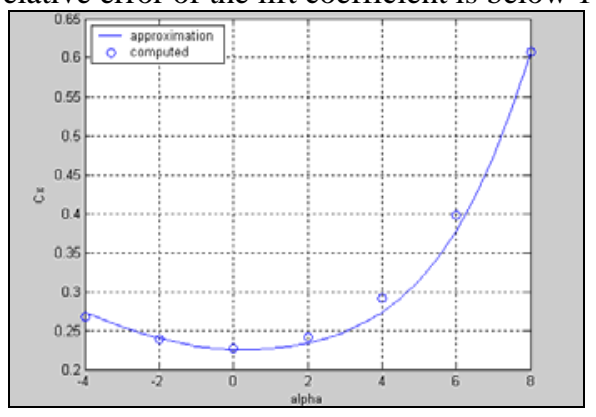

(a) Drag coefficient vs alpha

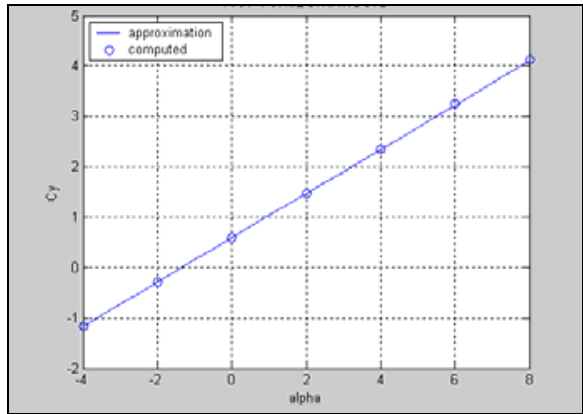

(b) Lift coefficient vs alpha

Figure 2. aerodynamics coefficient comparing between Surrogate model and original model

\section{CONCLUSION}

A method for predicting the aerodynamics coefficient of aircraft with arbitrary shape cross sections is propose based on boundary element method and Component Build-Up method. The results show a good agreement between computed data and wind tunnel data, which can satisfy the requirement of accuracy in concept design stage. A surrogate model is derived based on Latin Hypercube technique and Radial Basis Function, which do not reduce the calculation accuracy and improve the calculation efficiency. This method appears sufficient to predict aerodynamics coefficient of aircraft with arbitrary shape cross sections in concept design stage. However, the wing-body interference is considered the same as the body with circular section, which is lack of theory foundation and should be researched in future work.

\section{REFERENCES}

[1] Beall BW. Application of slender body theory to missiles with arbitrary shape cross sections . AIAA1986-0488

[2] Krieger RJ,Williams JE. Accuracy criteria for evaluating supersonic missile aerodynamic coefficient predictions . AIAA1981-1894

[3] Krieger RJ. An aerodynamic and signature shaping technique for developing advanced supersonic missile concepts. AIAA1982-373

[4] Sankar LN, Prichard D. Solution of transonic flow past rotor blades using the conservative full potential equation. AIAA 1985-5012

[5] Jenn AA, Williams JE. Preliminary aerodynamic design of arbitrary cambered missile bodies . AIAA1989-0528

[6] Bennett BK. Conceptual design synthesis tool for arbitrary-body missile . AIAA1997-2281

[7] Ji Xiuling, Zhang Taiheng, He Guanglin. Experiment on aerodynamic characteristics of loitering munitions with non-circular cross section. Journal of Nanjing University of Science and Technology(Natural Science), 2010, $01:$ 247-253

[8] Wei Jingbiao, Mi Zhihao, Sun Ruishen. Aerodynamic Characteristic Calculation of a Certain Noncircular Cross- Sections Dispense. Fire Control \&Command Control. 2003 28(5):56-58

[9] Chu Liang, Ma Dongli, Liu Zhongtie. Integrated design of aerodynamic and stealthy performance for missiles with non-circular cross section. Journal of Beijing University of Aeronautics and Astronautics, 2009,35 (10) : 1268-1273

[10] Yang Dequanm, Zhao Zhongcheng. Boundary element method and application, Beijing Institute of Technology press ,2002.9:30-45 
[11] JACK N.Nielsen, MISSILE AERODYNAMICS, McGRAW-HILL BOOK COMPANY NEWYORK, 1960

[12] Brown RM. Aerodynamic Coefficient Derivatives of Sonic Missiles via Slender Body Theory, Naval Research Laboratory Report 8203, January, 1979.

[13] Hess JL, Smith AMO. Calculation of Potential Flow About Arbitrary Bodies, Progress in Aeronautical Sciences Vol. 8, D. Kuchemann, Ed., Pergamon Press, Oxford, 1967.
[14] Anthony A.G, Steven F.W, Michael S.E. Overview of modern design of experiments methods for computational simulations. AIAA 2003649

[15] Marcelo J. Colaço George S. Dulikravich A Hybrid RBF Based Method for Highly Multidimensional Response Surfaces Using Scarce Data Sets AIAA 2008-5892

TABLE I. COMPARISON OF BOUNDARY ELEMENT METHOD AND HESS\&SMITH METHOD FOR APPARENT AREA

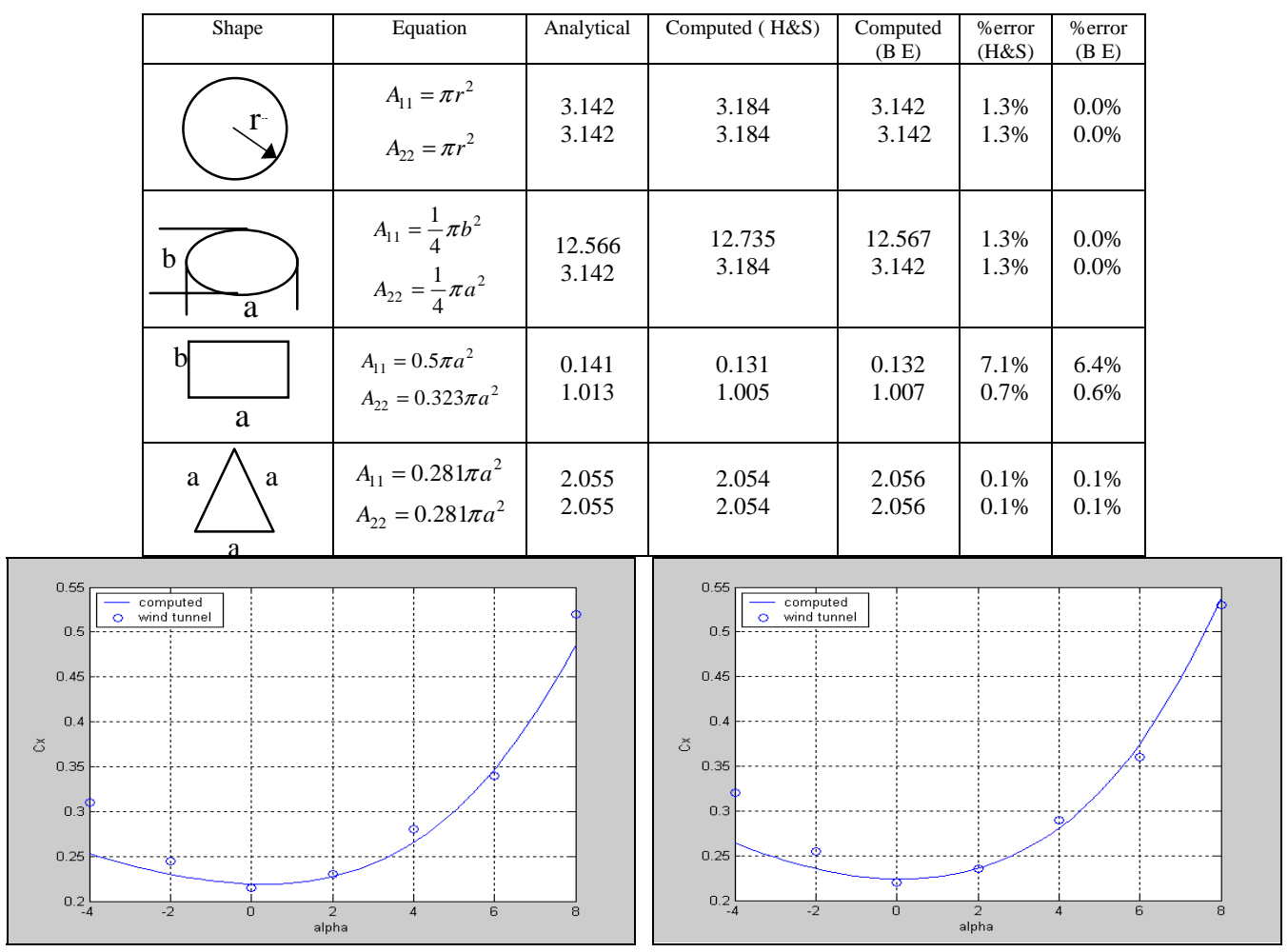

(a)Drag coefficient vs alpha of wing 1

(b)Drag coefficient vs alpha of wing 2

Figure 3. Drag coefficient vs alpha of two different wings

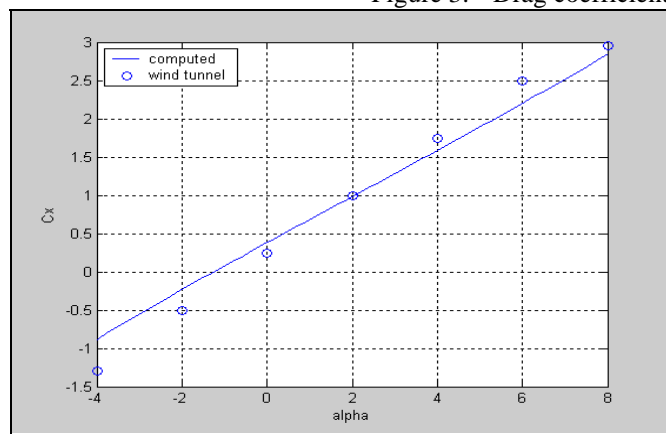

(a)Lift coefficient vs alpha of wing 1

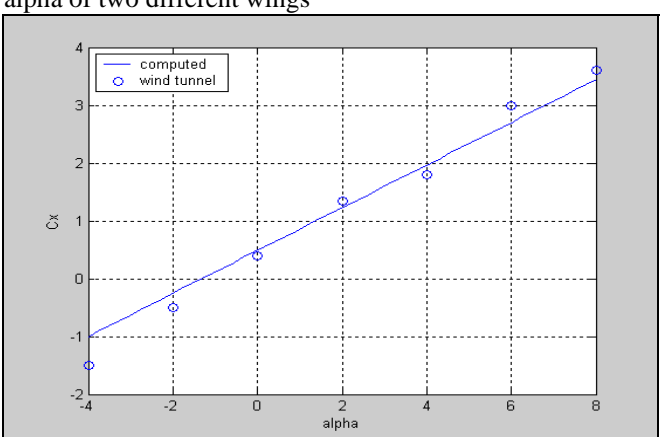

(b) Lift coefficient vs alpha of wing 2

Figure 4. Lift coefficient vs alpha of two different wing 